\title{
Noise Radar Technology as an Interference Prevention Method
}

\author{
Gaspare Galati and Gabriele Pavan \\ Department of Electronic Engineering, Tor Vergata University, Via del Politecnico, 1-00133 Rome, Italy \\ Correspondence should be addressed to Gaspare Galati; gaspare.galati@uniroma2.it
}

Received 6 June 2013; Accepted 6 July 2013

Academic Editor: Sandra Costanzo

Copyright (C) 2013 G. Galati and G. Pavan. This is an open access article distributed under the Creative Commons Attribution License, which permits unrestricted use, distribution, and reproduction in any medium, provided the original work is properly cited.

\begin{abstract}
In some applications, such as automotive and marine/navigation, hundreds of radars may operate in a small environment (e.g., a road complex or a strait) and in an allocated frequency band with limited width. Therefore, a compatibility problem between different radars arises that is not easily solved by time, frequency, space, or polarization diversity. The advent of fast digital signal processing and signal generation techniques makes it possible to use waveform diversity to solve this problem that will be exacerbated in the next future. Ideal waveforms for the diversity are supplied by Noise Radar Technology (NRT), whose application is promising in some military applications as well as in the civilian applications considered in this paper. In addition to being orthogonal as much as possible, the random signals to be transmitted have to satisfy requirements concerning side lobe level and crest factor, calling for novel, original design and generation processes.
\end{abstract}

\section{Introduction}

In spite of its military origin, dated back to the 1930s, radar technology is being more and more applied in the civilian domain for the safety of air, sea, and land traffic. In sea and road applications, this technology is entering the mass market with a significant yearly reduction of costs, mainly due to the larger and larger integration of the hardware and to the scale economy. For example in the automotive radar the factory cost has fallen by one order of magnitude (from hundreds to tens of dollars) in a few years. A similar situation is to be expected in the field of marine radar $[1,2]$, present in most vessels, as they (X-band radar) are mandatory for all passenger vessels and for all vessels above 500 tons GT (Gross Tonnage), with a double-band requirement (X-band radar + S-band radar) above 3000 tons GT. The allocated bands are 9.3 to $9.5 \mathrm{GHz}(\mathrm{X})$ and 2.9 to $3.1 \mathrm{GHz}$. Most marine radars use the simple and cheap magnetron transmitter, a 1940s technology, generating short pulses (from 50-80 ns in the short-range mode to 800-1000 ns in the long-range mode, up to $96 \mathrm{NM}$ ) and peak power from a very few $\mathrm{kW}$ to $12.5-25 \mathrm{~kW}$ (and $50 \mathrm{~kW}$ in coastal applications for Vessel Traffic Systems).

The large number of marine radars in a given area, simultaneously present above the horizon, makes the mutual interference a very likely event. For a general overview of potential solution to this problem, see [3, 4]. In marine radars the most used solution is based on their relatively short pulses that can be suppressed (video blanking or thresholding) because they are received asynchronously with respect to the transmission from the victim (the own) radar. Such a suppression causes tolerable detection losses for small targets and negligible losses for large targets.

As in many other radar applications, for example, in Air Traffic Control (ATC), solid-state transmitter technology is attractive for marine radars. It permits (i) the implementation of highly stable, coherent Rx-Tx chains compatible with Doppler processing, (ii) the transmission of sophisticated waveforms [5], (iii) a better availability, and (iv) the absence of high voltages. Unlike ATC radars that have been using solid state transmitters for over two decades, solid-state marine radars are appearing on the market just now, most of them, in the S-band. While maintaining approximately the same average transmitted power as magnetron transmitters, these new (or experimental) solid-state marine radars have three orders of magnitude larger duty cycle, that is, in the range of $10 \%-12 \%$. These long pulses (about 10 or 20 microseconds, in some cases up to 100 microseconds) are not compatible with the current interference suppression techniques. In 
the remaining part of this paper a new technique is proposed to solve this interference problem, whose general framework is synopsized in the appendix.

\section{Signal Requirements and Advantages of Noise Radar Technology (NRT)}

Mitigation techniques for interferences from similar radars can be based on diversity in (i) frequency, (ii) polarization, (iii) direction (antenna pattern adaptation), and (iv) waveform. In the marine radar context, as considered here, the limited allocated bandwidth (only $200 \mathrm{MHz}$ in X-band and the same in the S-band) and the use of standard antennae with fixed polarization prevent the use of (i), (ii), and (iii). Therefore a possible solution has to be based on waveform diversity, that is, the transmission (by the various radars in potential interference conditions) of pairwise orthogonal waveforms, such that the matched filter output is mainly due to the "own" waveform, with a noise floor due to the "other radars" waveforms. The large number of radars in busy maritime environments such as straits and sea areas around harbors, makes it necessary to exploit a large number of orthogonal waveforms, which can only be obtained by polyphase codes [3] or, as proposed here, by suitable processing of samples of random processes, leading to Noise Radar Technology.

The main requirements for a pair of signals with complex envelope $s_{i}(t)$ and $s_{j}(t)$ with $i, j=1, \ldots, M$, pulsewidth $T$, and the same power are defined by the following:

(i) Peak Side Lobe Ratio $(<-30 \mathrm{~dB})$ :

$$
\operatorname{PSLR}=\frac{\max _{i}\left(s_{i}\right)}{\max _{k}\left(m_{k}\right)},
$$

where $s_{i}=$ side lobe samples and $m_{k}=$ main lobe samples.

(ii) Crest Factor (C) or Peak-to-Average Ratio (PAR), that is, the peak amplitude of the waveform divided by the rms value of the waveform $s(t)$ :

$$
C=\frac{\max (|s(t)|)}{\sqrt{(1 / T) \int_{0}^{T}|s(t)|^{2} d t}} .
$$

(iii) Mean Envelope-to-Peak Power Ratio:

$$
\operatorname{MEPPR}=\frac{(1 / T) \int_{0}^{T}|s(t)|^{2} d t}{\max \left(|s(t)|^{2}\right)} .
$$

It results in $\mathrm{EPPR}=1 / \mathrm{C}^{2}$.

(iv) Normalized cross-correlation:

$$
r_{i j}(t)=\frac{\left|R_{i j}(t)\right|}{\left|R_{i(j)}(0)\right|},
$$

where $R_{i j}(t)=\int s_{i}^{*}(\theta) s_{j}(t+\theta) d \theta, i \neq j$; it measures the orthogonality, and the desired value is $r_{i j}(t)<-30 \mathrm{~dB}$. (v) Spectral band occupancy: sometimes this item is overlooked, especially when noise-like waveforms are concerned, but it is of paramount importance in most real-world radars; see [4] and the appendix.

Good candidate deterministic signals that satisfy the orthogonality requirements are the well-known "up" and "down" chirps (Linear-FM and Non-LFM) [3,5], but in this case only pairs of signals (not the needed multiplicity $M$ ) can be obtained. To obtain $M$ signals Costas codes, All top sequences can be considered, and polyphase codes represent a possible solution $[3,6,7]$. More recent research on orthogonal signals proposed the use of normal or interleaved OFDM techniques [8]. The main limitation of the OFDM approach is due to the nonconstant envelope of the signals, that is, MEPPR $<1$, which means that the transmitter does not work at its maximum power.

Another class of waveforms, that is, the phase noise signals [9], has two main advantages as compared to the signals introduced before. The former is the possibility to generate a large number of orthogonal signals, which is of great importance in the present case. The latter, applicable to defense applications (e.g., to the navigation radars of military vessels), is about the detectability; in fact they are random signals so they limit the detection, the identification, and the eventual spoofing of the signal. Such a limitation is of great importance in many military applications which require low detectability of the active system. Finally the MEPPR (and the crest factor) can be very close to the unity.

\section{Methods for Generating Noise Radar Signals}

For a phase noise signal the complex envelope can be written as

$$
s(t)=A \cdot \exp \{j \varphi(t)\} \cdot \operatorname{rect}_{T}(t),
$$

where $A$ is the constant amplitude, $\operatorname{rect}_{T}(t)$ is 0 outside the interval $[-T / 2,+T / 2]$ and 1 inside it (with $T$ being the pulse length), and $\varphi(t)$ is the phase process modulating the noise signal $s(t)$. In the following we present three methods to generate the phase noise signals highlighting their strengths and weaknesses.

3.1. Random Phase Modulation. In [9] Axelsson supposed for $\varphi(t)$ a zero-mean Gaussian process with root mean square (rms) $\sigma$ and a given power with density spectrum within the band $b$. He showed that the normalized autocorrelation function of the signal $s(t)$ can be written in a closed-form expression as

$$
R(\tau)=\exp \left\{-\sigma^{2}[1-\rho(t)]\right\}
$$

where $\rho(\tau)$ is the correlation coefficient of $\varphi(t)$. For example, $\rho(\tau)=\sin (\pi b \tau) /(\pi b \tau)$ for a constant spectrum within the band $b$. Of course, $R(\tau)$ depends on the bandwidth $b$, on the pulse length $T$, and on the rms phase fluctuation $\sigma$. The bandwidth $b$ is related to the width of the main peak and defines the range resolution. An increase of $T$, and 


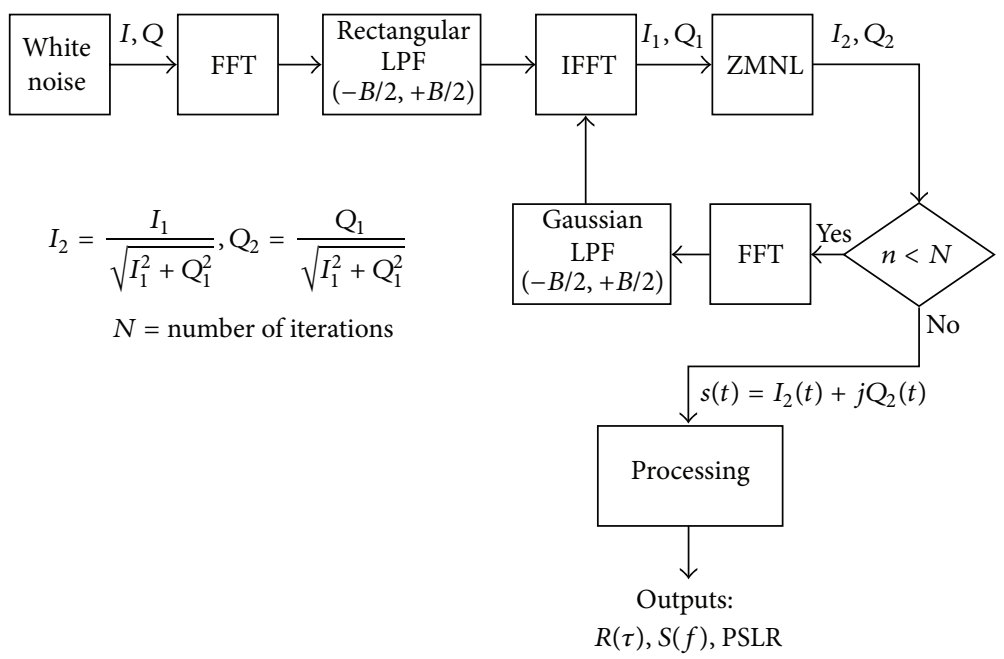

FIGURE 1: Block diagram of the iterative algorithm.

consequently of the compression ratio (the time-bandwidth product of the generated signal), causes a reduction in the range side lobe level, whereas the main lobe width remains fixed being independent of $T$. Finally the rms $\sigma$ has two different effects. The former is on the side lobe level: an increase of $\sigma$ causes a decrease of the side lobe level with improvement in the PSLR. The latter concerns the resolution. The rms value in fact establishes a connection between the bandwidth of the modulated signal and the bandwidth of the modulating signal. That is, when $\sigma$ increases the final bandwidth increases too. In [9] a simple relation between the rms bandwidth of the phase modulated signal $\left(B_{\mathrm{rms}}\right)$ and the rms bandwidth of the phase modulating noise $\left(b_{\text {rms }}\right)$ has been found:

$$
B_{\mathrm{rms}}=\sigma \cdot b_{\mathrm{rms}} \text {. }
$$

On the other hand, concerning the side lobe suppression, the expression of the autocorrelation function introduced in [9] would show a progressive improvement of the side lobe suppression as $\sigma$ increases. However the periodic nature of $\varphi(t)$ with a folding in the $[-\pi,+\pi]$ interval has been neglected in [9], and in reality the model can be used only for values of $\sigma$ significantly smaller than $\pi$. The Gaussian noise, used to modulate the signal phase, is to be compared with an uniform distribution in the range $[-\pi,+\pi]$ with a standard deviation of $(\pi / \sqrt{3}) \cong 1.8 \mathrm{rad}$. Therefore, if $\sigma$ is large $(\sigma>$ $(\pi / \sqrt{3}))$, the resultant phase is not Gaussian distributed and the mathematical formulation introduced in [9] does not apply. On the other hand, in connection with a potential real application, it would be better to generate the signal through a white Gaussian process with its in phase and in quadrature components $(I, Q)$ that are band limited as desired. This is described in the following.

3.2. An Iterative Algorithm to Generate Phase Noise Signals. To control the spectral width and to reduce the Peak Side Lobe Ratio (PSLR) of the generated phase noise signals, we propose an iterative algorithm based on alternative projections in frequency and in time domain (Figure 1). The input to the algorithm is a zero-mean white complex Gaussian process $(I+j Q)$. The filtering is implemented in frequency domain. First the input is band limited using a rectangular Low Pass Filter of band $B$. The output from this filter is projected in time domain by IFFT, and the complex signal $\left(I_{1}, Q_{1}\right)$ is amplitude limited using a Zero-Memory-NonLinearity $(Z M N L)$. The output in time $\left(I_{2}, Q_{2}\right)$ is reprojected in frequency and filtered through a Gaussian LPF of band $B$. The output is again re-projected in time, and the procedure is iterated for a predefined value of $N$. Typically to achieve the optimum (minimum) PSLR, some tens of iterations are needed (it depends on the input white noise sequence).

Considering for ZMNL a hard limiter, that is,

$$
I_{2}=\frac{I_{1}}{\sqrt{I_{1}^{2}+Q_{1}^{2}}}, \quad Q_{2}=\frac{Q_{1}}{\sqrt{I_{1}^{2}+Q_{1}^{2}}}
$$

the signal $\left(I_{2}, Q_{2}\right)$ shows a unitary amplitude and its randomness is only due to the random phase of the signal. Averaging different autocorrelations we have computed the mean normalized autocorrelation and its standard deviation (Figure 2 for BT $=10000$ ).

Varying the compression ratio up to 30000 , in Figure 3 the mean PSLR is shown.

To reach PSLR lower than $-30 \mathrm{~dB}$, compression ratios over 5000 are needed. However for BT $=30000$ the PSLR is $-36 \mathrm{~dB}$, less, for example, than the one obtained with NonLFM using Hamming weighting $(-42.8 \mathrm{~dB})$. Figure 4 shows the mean spectrum for BT $=10000(B=100 \mathrm{MHz})$. It shows a good control of the spectral width due to the rectangular LPF. Finally in Figure 5 the cross-correlation (it measures the orthogonality) for a pair of generated phase noise signals is shown with $\mathrm{BT}=10000$. It is lower than $-30 \mathrm{~dB}$ (as compared with the same compression ratio, for a pair of up and down chirps the cross-correlation is $-43 \mathrm{~dB}$ for LFM and $-40 \mathrm{~dB}$ for Non-LFM). 


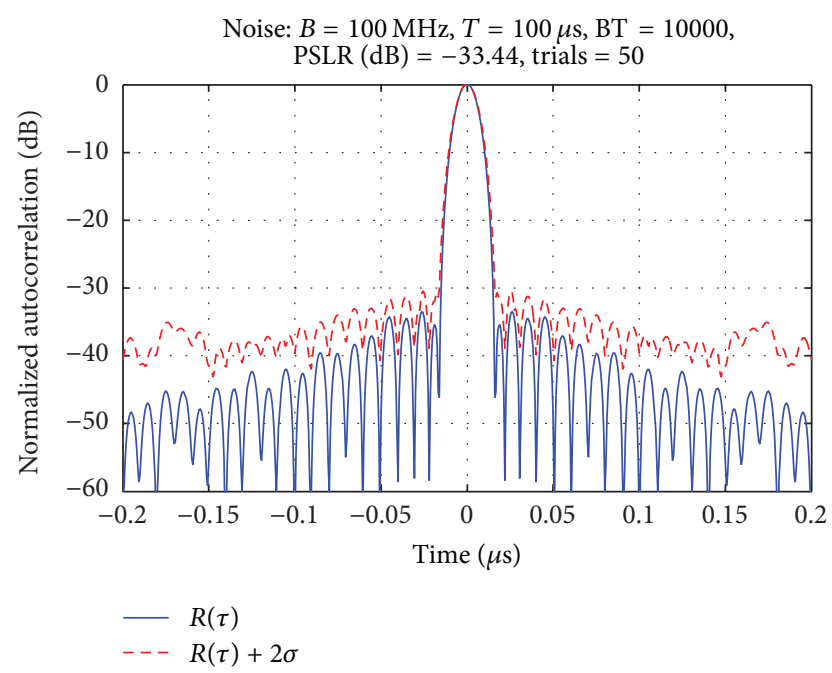

FIGURE 2: Mean normalized autocorrelation (obtained averaging 50 different autocorrelations). Dashed line: fluctuation at $2 \sigma$ ( $\sigma$ denotes the standard deviation). Compression ratio $\mathrm{BT}=10000$, and the $\mathrm{ZMNL}$ is a hard limiter.

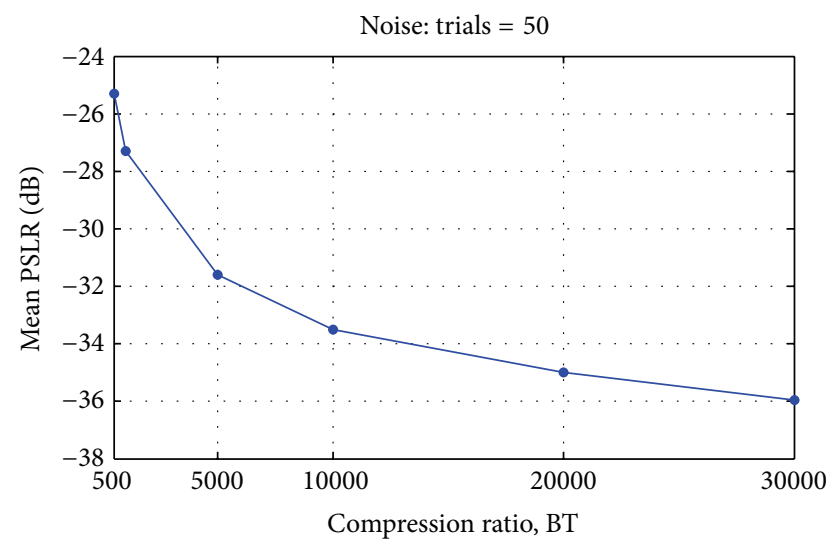

Figure 3: Mean PSLR (obtained averaging 50 different autocorrelations) versus the compression ratio BT, considering for ZMNL a hard limiter.

From the database of generated waveforms [10] a part is selected after analysis of the compliance with the required characteristics in terms of spectral occupancy and autocorrelation and cross-correlation functions. In order to further reduce the side lobe level (which is likely needed, especially for smaller values of the compression ratio BT), a mismatched filtering in reception may be needed $[11,12]$. The filter coefficients must be computed for every selected waveform and associated with it for the ensuing usage. While permitting a significant suppression of the range side lobes, this technique calls for increased computation costs (the mismatched filter is typically three times longer than the matched filter) and larger memory requirements.

3.3. Closed Form by Inversion. A different, closed form generation approach is based on the following considerations.

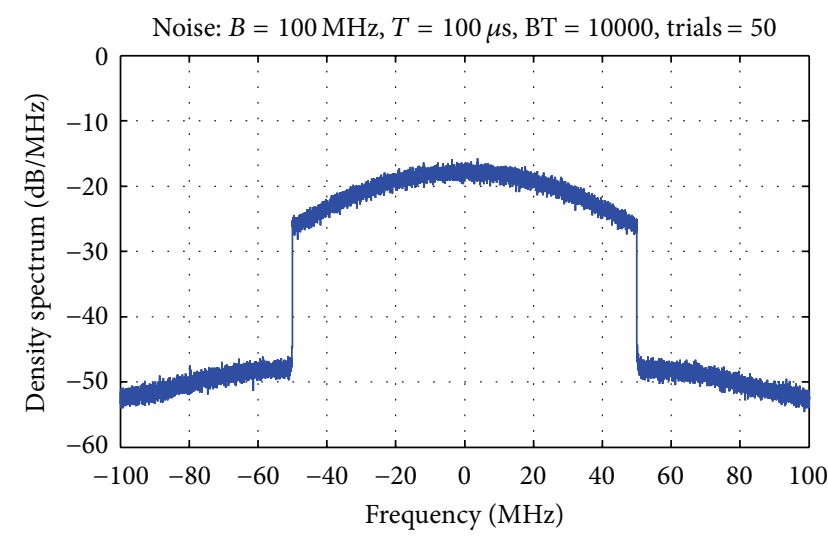

FIGURE 4: Mean density spectrum (obtained averaging 50 different spectra) for a pair of phase noise signals. Compression ratio 10000, considering for ZMNL a hard limiter.

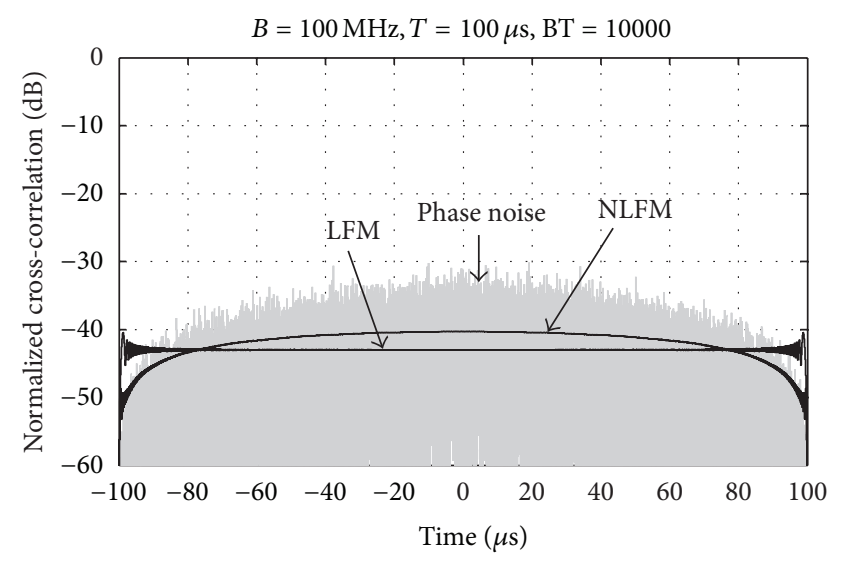

FIGURE 5: Normalized cross-correlation for a pair of generated noise phase signals by the iterative algorithm considering for ZMNL a hard limiter. Compression 10000.

For a real Gaussian process Van Vleck and Middleton [13] have shown that the autocorrelation coefficient $\left(R_{t}\right.$ with $t=$ $t_{2}-t_{1}$ ) of the output from a hard limiter is related with the input autocorrelation coefficient (here denoted $r$ ) by the wellknown arcsine law:

$$
R_{t}=\frac{2}{\pi} \cdot \arcsin (r)
$$

Considering a complex Gaussian process, the correlation $R_{t}$ after the hard limiter, that is, between

$$
\begin{aligned}
& \frac{z_{1}^{*}}{\left|z_{1}\right|}=\frac{x_{1}-j y_{1}}{\sqrt{x_{1}^{2}+y_{1}^{2}}}, \\
& \frac{z_{2}}{\left|z_{2}\right|}=\frac{x_{2}+j y_{2}}{\sqrt{x_{2}^{2}+y_{2}^{2}}},
\end{aligned}
$$


is

$$
\begin{aligned}
R_{t} & =E\left\{\frac{z_{1}^{*} z_{2}}{\left|z_{1}\right|\left|z_{2}\right|}\right\} \\
& =E\left\{\frac{x_{1} x_{2}+y_{1} y_{2}+j\left(x_{1} y_{2}-x_{2} y_{1}\right)}{\sqrt{x_{1}^{2}+y_{1}^{2}} \sqrt{x_{2}^{2}+y_{2}^{2}}}\right\}=u+j v,
\end{aligned}
$$

where $E\{\cdot\}$ is the statistical mean operator. Supposing a symmetrical power density spectrum with respect to the origin, the correlation is real and $v=0$. Equation (11) has been evaluated, and it results in

$$
R_{t}=b_{0} r+\sum_{n=1}^{\infty} b_{n} \cdot r^{2 n+1}
$$

with

$$
b_{n}=\frac{(2 n-1)^{2}}{4 n(n+1)} \cdot b_{n-1}, \quad b_{0}=\frac{\pi}{4}, \quad n=1,2,3, \ldots
$$

Then $R_{t}$ can be expressed as a sum of odd powers of $r$, where the coefficients $b_{n}$ are very similar to those evaluated for the arcsine law:

$$
b_{n}=\frac{(2 n-1)^{2}}{2 n(2 n+1)} \cdot b_{n-1}, \quad b_{0}=\frac{2}{\pi}, \quad n=1,2,3, \ldots
$$

Figure 6 shows $R_{t}$ versus the input correlation $r$ for real and complex Gaussian process. Inverting (12) it is possible to predistort the input autocorrelation to the hard limiter to obtain a desired $R_{t}$.

In such a way the requirements of Sections I and II can be met with no need for iterations. In fact, (a) the output autocorrelation is chosen in order to satisfy the PSLR requirement and the spectral band requirement; (b) the MEPPR requirement is satisfied by the hard limiter, and (c) the orthogonality is obtained by the randomness of the white Gaussian input sequence; and may be enhanced by proper choices of the generated output sequences.

\section{An Architecture for Next Generation Marine Radar Based on NRT}

The general architecture of a proposed, novel class of solidstate, coherent, NRT-based marine radar is shown in Figure 7, from [10]. Basically, this is a coherent radar transmitting preregistered pseudorandom signals and using a correlation receiver (or a mismatched, range-side lobe suppression receiver). In order to reduce the interference level between these marine radars exploiting the orthogonality of the transmitted waveforms, each radar must have a large number of them (order of hundreds of millions) stored in a mass memory; see Figure 7.

These waveforms are result of a generating procedure shown in Figure 1, as explained before. Thanks to this procedure, all of them have a crest factor close to the unity and their power spectral density (PSD) respects the spectral

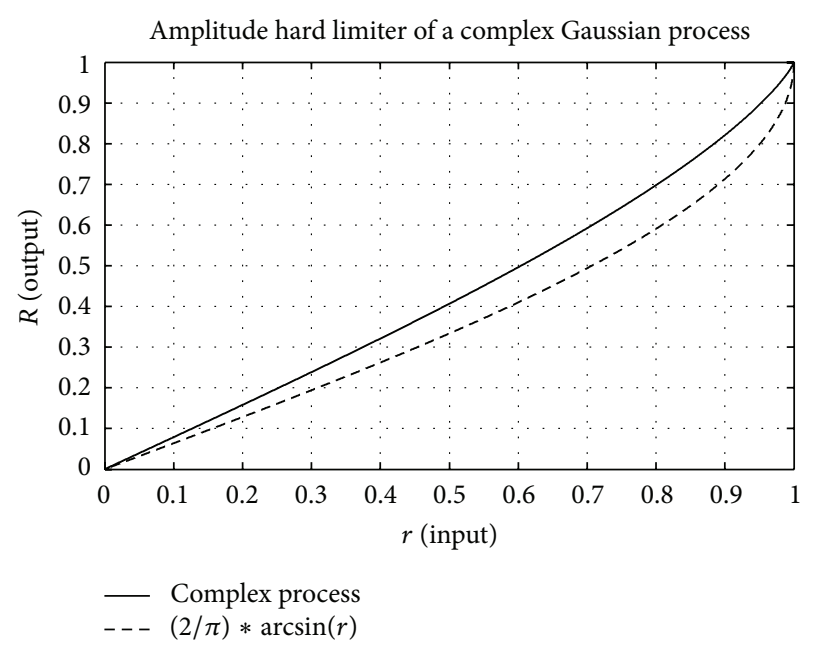

FIGURE 6: Output autocorrelation $(R)$ from a hard limiter versus the input autocorrelation $(r)$.

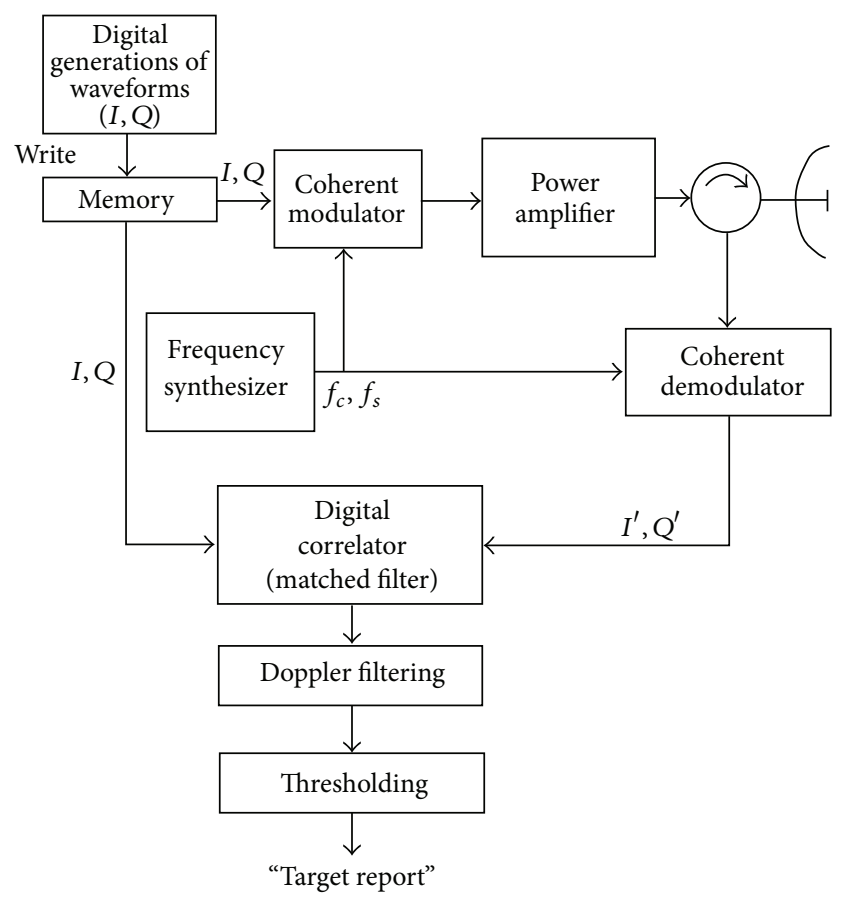

FIGURE 7: General architecture of solid-state, coherent, NRT-based marine radar.

mask of the international regulations (see the appendix). For instance, let us consider a waveform, with a bandwidth of $80 \mathrm{MHz}$ and a centre frequency of $9410 \mathrm{MHz}$, that is, a spectrum from 9.37 to $9.45 \mathrm{GHz}$ with EIRP per $\mathrm{MHz}$ equal to $75 \mathrm{dBm}$. Outside the interval (the "Necessary Band") from 9.37 to $9.45 \mathrm{GHz}$ its PSD will have to decay at $20 \mathrm{~dB} / \mathrm{dec}$, arriving at $35 \mathrm{dBm}$ EIRP below $9.17 \mathrm{GHz}$ and above $9.64 \mathrm{GHz}$. Only a part of the generated waveforms, that is, those with an acceptable PSR, is selected to be stored in the mass memory.

As in solid-state marine radar using deterministic waveforms and pulse compression, $[14,15]$, in the proposed marine 
noise radar three sets of length will be used: a short pulse for sort range, a medium-duration waveform for medium range, and a long waveform for the maximum range of $96 \mathrm{NM}$.

\section{Plans for Future Work and Conclusion}

It has been recognized (see for instance [14]) that the long pulses of solid-state marine radar will create formidable problems of electromagnetic comparability to (a) traditional magnetron radar as well as to (b) other solid-state marine radars. Usage of pseudorandom, orthogonal signals will alleviate case (b) by a figure close to the value of the compression factor (the BT product). Further analysis is needed for case (a). For example, in [14] it is stated that "characteristic pulsed interference was observed, caused by the long pulses disabling pulse cancellation logic in the magnetron radar. Such interference on established radar systems raised concerns to the system's further development ...." and " $A$ novel 3 pulse waveform has been designed and tested for solid-state marine radar. The waveform's spectral and range performance are excellent,... However the interference effects that such a radar might cause on existing marine radars may be catastrophic. Some of these effects could be reduced by techniques that were investigated, however no techniques were found that gave confidence to proceed with a solid state marine radar product based on this waveform." The damaging signals are of two types: direct (antenna to antenna) and indirect or bistatic clutter (antenna to reflecting surfaces to antenna). Summing up, further analysis, simulation, and trials are needed to establish the cost-effectiveness of solid-state $\mathrm{S}$ - and $\mathrm{X}$-bands marine radars to be used in crowded environments as well as the electromagnetic compatibility between them and, presently more important, with legacy marine radar. The Noise Radar Technology is a promising tool to solve these problems.

\section{Appendix}

\section{Interference in Marine Radar}

Recommendations and rules for avoiding and/or mitigating the interferences related to radar systems have been issued by the International Electro Technical Committee [16] and by the International Telecommunication Union [17, 18]. Generally, distinction is made between (i) the "Necessary Band," related to the shape and modulation of the radar signal as designed to satisfy the radar performance, (ii) the "Out of Band" (OoB) domain that contains the spectral residuals of the modulation process for the radar signal, and (iii) the "spurious" domain that contains the harmonic, parasitic, intermodulation and frequency conversion effects. Methods are suggested to compute the Necessary Band from the pulse length and its rise and fall time or from the frequency span in the cases of linear or nonlinear frequency modulation (chirp signals). Roughly, the Necessary Band for a simple rectangular pulse of duration $T$ is about $1.8 / T$, and for a linear chirp of frequency deviation $\Delta f$ is about $2 \Delta f$. A $-40 \mathrm{~dB}$ band is defined in the radar emission mask; in some significant cases, it results in $B_{-40}=2.5 B_{\text {Necessary }}$ and the transition between them has a $20 \mathrm{~dB} /$ decade roll-off.

\section{References}

[1] A. Bole, B. Dineley, and A. Wall, Radar and ARPA Manual, Elsevier, New York, NY, USA, 2nd edition, 2005.

[2] J. N. Briggs, Target Detection by Marine Radar, vol. 16 of IEE Radar, Sonar and Navigation Series, The Institute of Electrical Engineers, London, UK, 2004.

[3] S. Harman, "Spectral sharing with radar," in Principles of Waveform Diversity and Design, M. Wicks, M. Wicks, E. Mokole, S. Blunt, R. Schneible, and V. Amuso, Eds., Part 6: Spectrum Management, Chapter 35, SciTech, Edison, NJ, USA, 2010.

[4] J. T. Ascroft, "A study into techniques for improving radar spectrum utilization," Project AY, 4490, 2004, http://citeseerx .ist.psu.edu/viewdoc/download?doi=10.1.1.127.6876\&rep=rep1\& type $=$ pdf.

[5] N. Levanon and E. Mozeson, Radar Signals, IEEE Press, WileyInterscience, Jhon Wiley \& Sons, New York, NY, USA, 2004.

[6] J. P. Costas, "A study of detection waveforms having nearly ideal range-doppler ambiguity properties," Proceedings of the IEEE, vol. 72 , no. 8, pp. 996-1009, 1984.

[7] W. O. Alltop, "Complex sequences with low periodic correlations," IEEE Transactions on Information Theory, vol. 26, no. 3, pp. 350-354, 1980.

[8] P. van Genderen, O. A. Krasnov, Z. Wanga, and R. F. Tigrek, "Some OFDM waveforms for a fully polarimetric weather radar," International Journal of Microwave and Wireless Technologies, vol. 4, no. 02, pp. 163-169, 2012.

[9] S. R. J. Axelsson, "Noise radar using random phase and frequency modulation," IEEE Transactions on Geoscience and Remote Sensing, vol. 42, no. 11, pp. 2370-2384, 2004.

[10] G. Galati, "Radar coerente," Patent Application N. RM, 2013 A000290, Roma, Italy, 2013.

[11] A. S. Mudukutore, V. Chandrasekar, and R. Jeffrey Keeler, "Pulse compression for weather radars," IEEE Transactions on Geoscience and Remote Sensing, vol. 36, no. 1, pp. 125-142, 1998.

[12] H. Urkowitz and N. J. Bucci, "Doppler-Tolerant range sidelobe suppression for meteorological radar," IEEE Transactions on Geoscience Electronics, vol. 4, pp. 206-208.

[13] J. H. Van Vleck and D. Middleton, "The cpectrum of clipped noise," Proceeding of the IEEE, vol. 54, pp. 2-19, 1966.

[14] S. Harman, "The performance of a novel Three-Pulse radar waveform for marine radar systems," in Proceedings of the 5th European Radar Conference (EuRAD '08), pp. 160-163, Amsterdam, The Netherlands, October 2008.

[15] A. Nelander and Z. Tóth-Pál, "Modular system design for a new S-band marine radar," in Proceedings of the International Radar Conference Surveillance for a Safer World (RADAR '09), Bordeaux, France, December 2009.

[16] International Electro Technical Commission, "Shipborne radar," IEC, 62388 Ed. 1. 0, 2007.

[17] ITU International Telecommunication Union, "Unwanted emission in the out of band domain," Recommendation ITUR SM, 1541, 2001.

[18] ITU International Telecommunication Union, "Efficient use of the radio spectrum by radar stations," Recommendation ITU-R M, 1327-1, 1998-2003. 

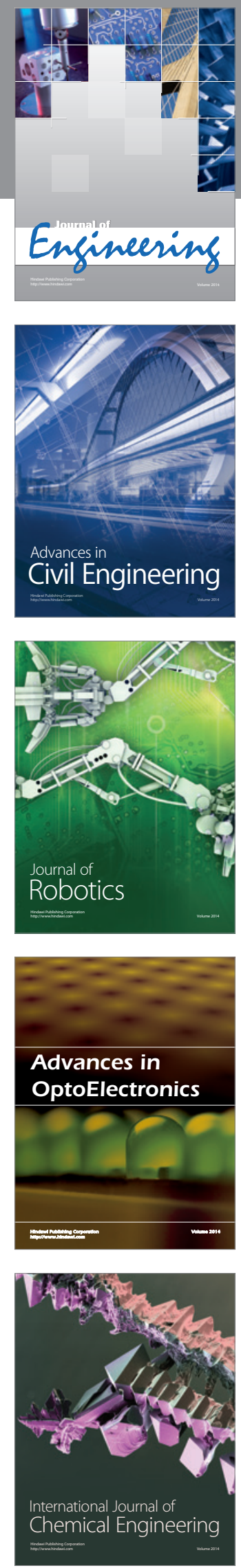

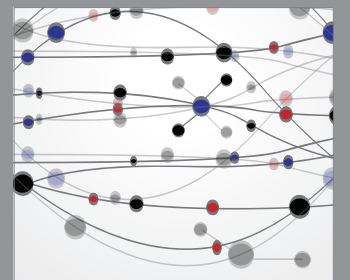

The Scientific World Journal
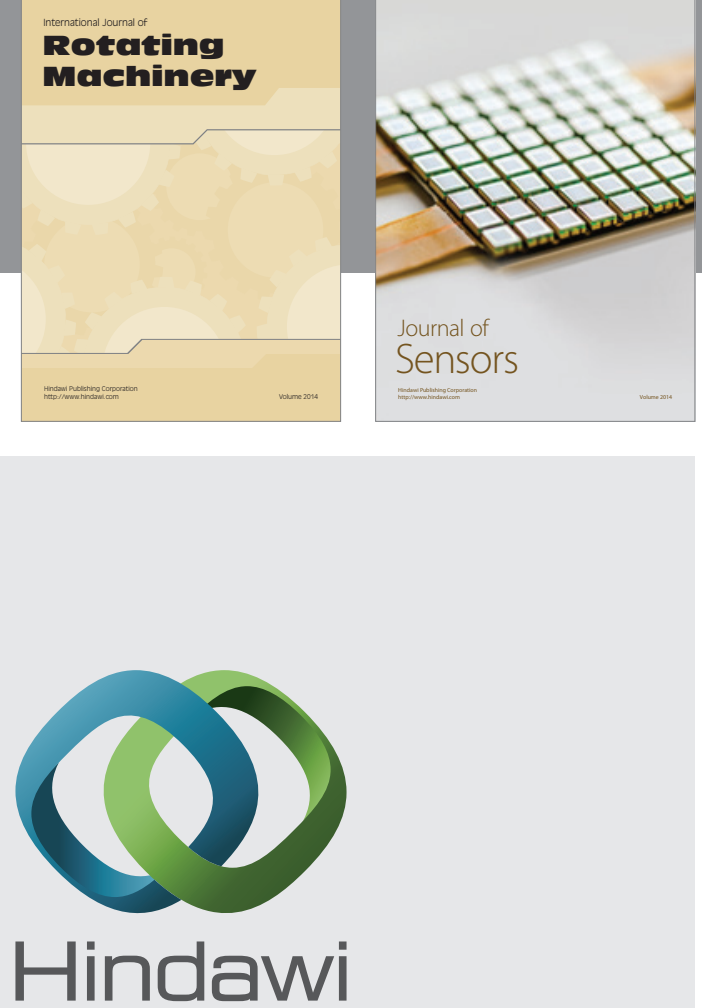

Submit your manuscripts at http://www.hindawi.com
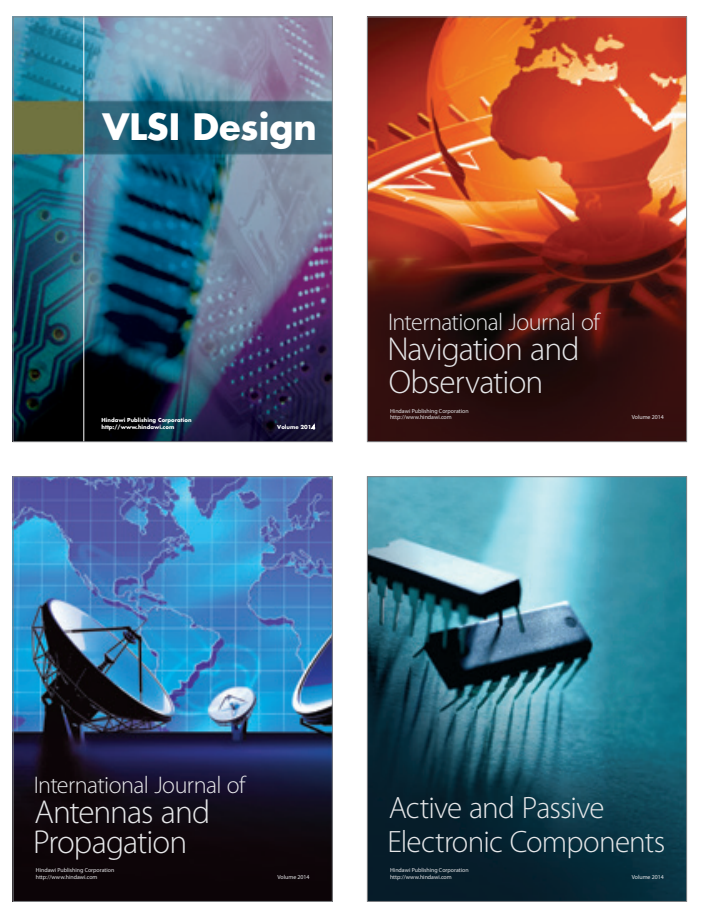
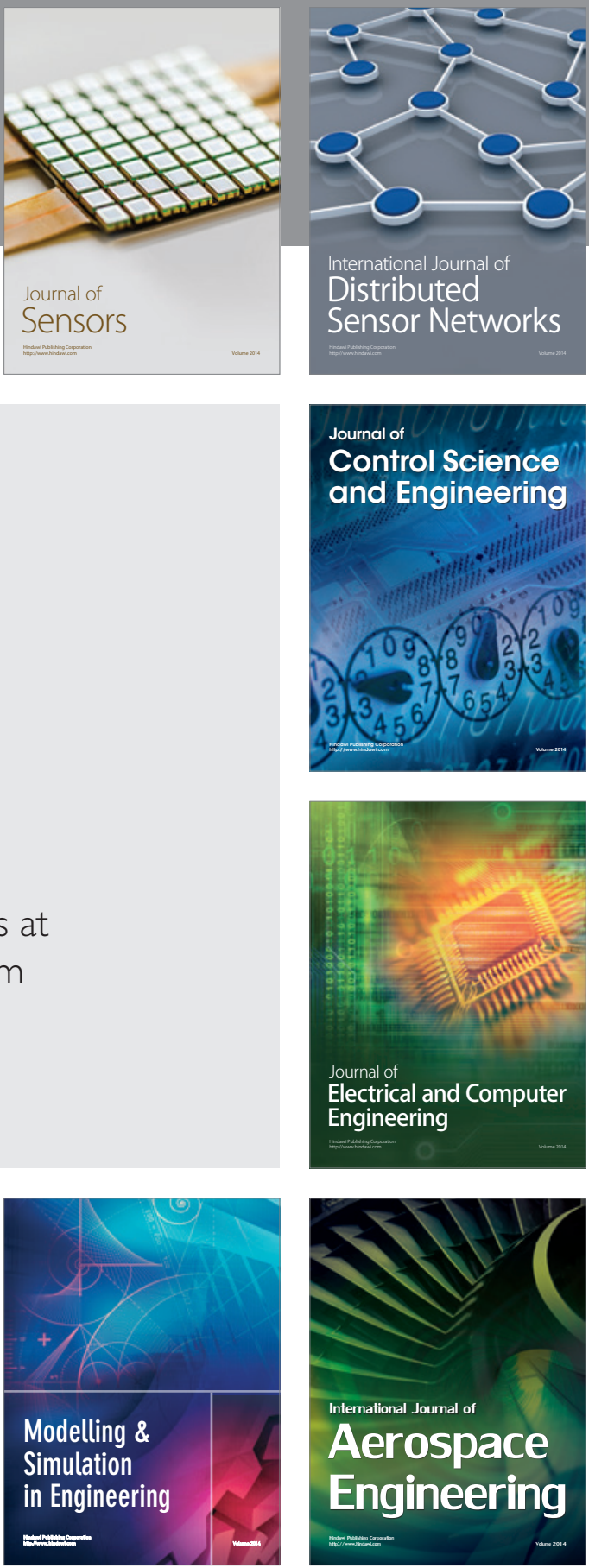

Journal of

Control Science

and Engineering
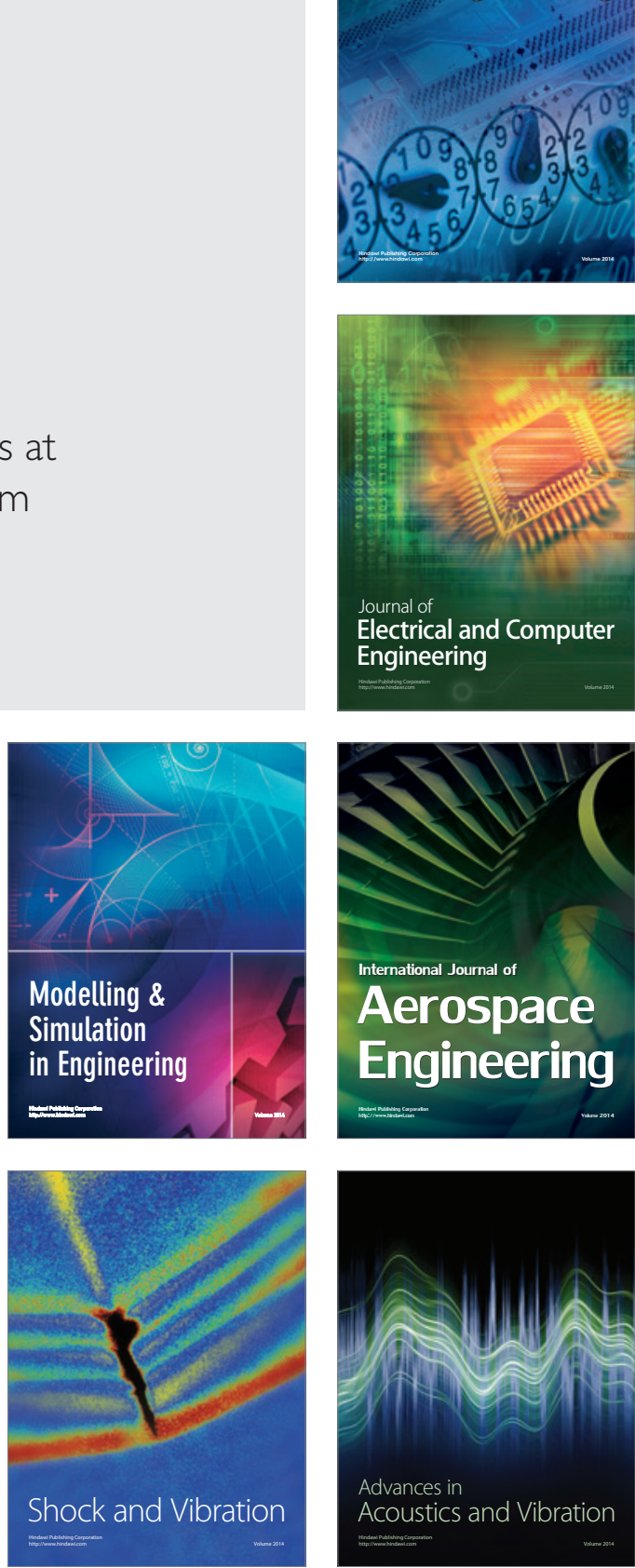